\title{
Analysis of counting measurements on narrowband frequency up-converted single photons and the influence of heralding detector dead time
}

\author{
Jaromír Fiurášek, ${ }^{1}$ Christoph Baune, ${ }^{2}$ Axel Schönbeck, ${ }^{2}$ and Roman Schnabel ${ }^{2,3}$ \\ ${ }^{1}$ Department of Optics, Palacký University, 17. listopadu 12, 77146 Olomouc, Czech Republic \\ ${ }^{2}$ Institut für Gravitationsphysik, Leibniz Universität Hannover and Max-Planck-Institut für Gravitationsphysik \\ (Albert-Einstein-Institut), Callinstrasse 38, 30167 Hannover, Germany \\ ${ }^{3}$ Institut für Laserphysik und Zentrum für Optische Quantentechnologien, Universität Hamburg, \\ Luruper Chaussee 149, 22761 Hamburg, Germany
}

(Received 23 October 2014; published 20 January 2015)

\begin{abstract}
We present a theoretical model of photon counting measurements on conditionally generated narrowband single-photon states that are produced via cavity-enhanced spontaneous parametric down-conversion, then frequency up-converted from the telecom wavelength of $1550 \mathrm{~nm}$ to the visible wavelength of $532 \mathrm{~nm}$. The highly nonclassical character of the up-converted states is certified by a quantum non-Gaussianity witness that is determined from coincidence measurements with single-photon detectors in a Hanbury-Brown-Twiss configuration. We find our model in good agreement with the experimental data, and we investigate a useful effect caused by the dead time of the trigger detector, whose clicks herald conditional preparation of the single-photon state. Due to the dead time, a click of the trigger detector excludes the possibility of a trigger event at a certain preceding time interval, during which the measured idler beam is thus projected onto a vacuum state. Due to quantum correlations between signal and idler beams, this reduces the multiphoton contributions in the conditionally generated state of the signal beam and accordingly increases the value of the quantum non-Gaussianity witness. We also show that spurious heralding detections due to after-pulsing can be suppressed by accepting a click of the trigger detector only if its distance from a previous click of this detector exceeds a certain suitably chosen threshold.
\end{abstract}

DOI: 10.1103/PhysRevA.91.013829

PACS number(s): 42.50.Ar, 42.50.Dv

\section{INTRODUCTION}

Conditioning on single-photon detection represents a very versatile and powerful tool in optical quantum state engineering and quantum information processing. Single-photon states can be conditionally prepared by detecting an idler photon that is part of a correlated photon pair, generated in the process of spontaneous parametric down-conversion [1-4]. Furthermore, approximate coherent superpositions of coherent states can be prepared by conditionally subtracting a photon from squeezed vacuum states [5-7]. These techniques can be extended to more sophisticated protocols such as the preparation of optical qubits encoded into superpositions of squeezed vacuum and single-photon states [8], implementation of noiseless quantum amplifiers [9-12], continuous-variable entanglement distillation [13,14], tests of quantum commutation relations [15], or generation of a resource state for emulation of quantum qubic nonlinearity [16].

Experiments involving photon counting on continuouswave squeezed light produced in an optical parametric amplifier (OPA) operating below threshold are of particular interest as the optical cavity ensures a well-defined transversal spatial optical mode, which facilitates mode matching with local oscillators when the states are characterized by homodyne detection. During recent years, various schemes of this kind were investigated both theoretically and experimentally [4,6,7,17-23]. A specific feature of these experiments is that due to the narrow bandwidth of the OPA cavity the temporal width of wave packets that contain the conditionally generated states is much larger than the resolution of the single-photon detectors, and the optimal wave packet may have a nontrivial shape. This platform thus allows for temporal multiplexing [23] and time-separated two-photon subtraction [22], which greatly increases the flexibility of quantum state engineering.

Recently, we have experimentally demonstrated frequency up-conversion [24-30] of conditionally generated singlephoton states from the telecom wavelength of $1550 \mathrm{~nm}$ to the visible wavelength of $532 \mathrm{~nm}$ [31]. The single-photon states were generated by detection of idler photons from a continuous-wave two-mode squeezed vacuum state, and the up-converted signal photons were characterized by photon counting measurements in a Hanbury-Brown-Twiss configuration. In that experiment we utilized a witness of quantum non-Gaussianity $[32,33]$ to certify the highly nonclassical character of the up-converted state. Together with our previous experiments on frequency up-conversion of coherent and squeezed states [34,35], we confirm that quantum upconversion can be highly efficient and preserves quantum coherence and nonclassical properties of the states. Quantum up-conversion therefore provides a suitable bridge between near-infrared telecom wavelengths optimal for long-distance transmission of quantum information and shorter wavelengths suitable for light-matter coupling, quantum memories, and efficient detection of single photons by silicon avalanche photodiodes [26-30].

In this paper, we present a detailed theoretical analysis of the experiment in Ref. [31], which fully accounts for the temporal structure of quantum correlations of continuouswave two-mode squeezed states. The theoretical predictions are found to be in a very good agreement with the experimental data, and our calculations reveal that the dead time $t_{D}$ of the trigger single-photon detector plays an important role [36-38]. Remarkably, we find that the dead time helps to increase the 
value of the quantum non-Gaussianity witness. The underlying physical mechanism behind this phenomenon is that a click of the trigger detector at time $t_{0}$ indicates that the trigger detector did not detect any photon in the time interval $\left[t_{0}-t_{D}, t_{0}\right]$, because otherwise it would be blind and could not have registered a photon at $t_{0}$. The trigger detector thus projects the idler beam onto a vacuum state during the time interval $\left[t_{0}-t_{D}, t_{0}\right]$. This effect was originally studied in connection with measurements of intensity correlation functions with time-to-amplitude converters [36-38]. Here we show that due to the quantum correlations between signal and idler beams the dead-time-induced projection on vacuum of the idler beam reduces the multiphoton contributions in the conditionally generated state of the signal beam, which makes it more easily detectable as a quantum non-Gaussian state by the witness that we employ.

The rest of the paper is organized as follows. In Sec. II we review the concept of quantum non-Gaussian states and define the quantum non-Gaussianity witness that is employed in our study. The experimental setup is described in Sec. III. In Sec. IV we present a simple single-mode model of the experiment, which allows us to explain our method to calculate the probabilities of observations of various coincidence clicks of the single-photon detectors, which is based on a Gaussian state formalism and the Husimi $Q$ function. In Sec. V we extend this model to a full multimode description that accounts for temporal quantum correlations between the signal and idler beams. In short, we discretize relevant time windows into a large number of narrow time slots, and associate an individual mode having a rectangular temporal profile with each slot. We calculate a multimode covariance matrix describing a Gaussian state of these modes prior to any detection and we then determine the probabilities of coincidence clicks following the procedure outlined in Sec. IV. Results of numerical calculations are discussed in Sec. VI, and Sec. VII contains a brief summary and conclusions.

\section{QUANTUM NON-GAUSSIAN STATES}

We say that a quantum state $\rho$ is quantum non-Gaussian if it cannot be expressed as a statistical mixture of Gaussian states [32]. A quantum non-Gaussian state cannot be prepared using only Gaussian coherent and squeezed states of light together with linear optics, and some higher-order nonlinearity has to be involved in its generation. In the present case this nonlinearity is provided by single-photon detection on the idler beam. Traditionally, the negativity of the Wigner function has been used to reveal the highly nonclassical character of quantum states of light. However, the negativity is sensitive to losses, and for instance a mixture of vacuum and single-photon state $p|1\rangle\langle 1|+(1-p)| 0\rangle\langle 0|$ has a positive Wigner function if $p<0.5$. Nevertheless, it can be shown that the state is quantum non-Gaussian for any $p>0$ [32]. Several witnesses of quantum non-Gaussianity have been proposed recently [32,39-42] and experimentally tested [33,42-44]. Here we employ a witness based on probabilities of vacuum and single-photon states $p_{0}$ and $p_{1}[32,33]$, which is particularly suitable for the certification of the highly nonclassical character of approximate single-photon states in the presence of losses or inefficient detection.
The witness of quantum non-Gaussianity is defined as a suitable linear combination of the vacuum and single-photon probabilities,

$$
W=\max _{a<1}\left[p_{1}+a p_{0}-W_{G}(a)\right]
$$

where $W_{G}(a)$ denotes the maximum of $p_{1}+a p_{0}$ over all Gaussian states, $a<1$ is a real parameter and a maximum over all $a$ is taken. Quantum non-Gaussianity of the state is certified if $W>0$. As shown in Ref. [33], $W_{G}(a)$ is given by the following expression,

$$
W_{G}(a)=\frac{e^{-d^{2}[1-\tanh (s)]}}{\cosh ^{3}(s)}\left[d^{2}+a \cosh ^{2}(s)\right],
$$

where $d^{2}=\left(e^{4 s}-1\right) / 4$ and

$$
a=\frac{e^{2 s}\left(3-e^{2 s}\right)}{1+e^{2 s}} .
$$

The maximization over $a$ in Eq. (1) can be equivalently replaced by maximization over $s$, which leads to a transcendental equation for the optimal $s$,

$$
p_{0} \cosh (s) e^{e^{2 s} / 2}-\sqrt{e}=0 .
$$

Once the optimal $s$ is found, the corresponding values of $a$ and $W_{G}(a)$ can be calculated using Eqs. (2) and (3), and the non-Gaussianity witness can be determined by evaluating $p_{1}+a p_{0}-W_{G}(a)$.

\section{EXPERIMENTAL SETUP}

The experimental setup is depicted in Fig. 1 [31]. The main laser source was a Nd:YAG laser with $2 \mathrm{~W}$ output power, that was frequency doubled to $532 \mathrm{~nm}$ in a $\mathrm{LiNbO}_{3}$-based second harmonic generator (not shown in the figure). A major fraction of the $532 \mathrm{~nm}$ field pumped a monolithic nondegenerate, doubly resonant optical parametric oscillator (OPO) above threshold, providing up to $200 \mathrm{~mW}$ at $810 \mathrm{~nm}$ to pump the quantum up-converter (QUC). A smaller fraction of the $532 \mathrm{~nm}$ field was used as the pump field for another nonlinear cavity, which was operated below threshold and generated twin photons at $810 \mathrm{~nm}$ and $1550 \mathrm{~nm}$ in a cavity enhanced spontaneous parametric down-conversion process (SPDC). The $810 \mathrm{~nm}$ heralding photons passed a Fabry-Perot filter cavity (FC) to suppress uncorrelated modes. The transmitted photons were detected with a Si-APD (Perkin Elmer SPCM-AQRH-13). The $532 \mathrm{~nm}$ photons were split on a balanced beam splitter and detected with Si-APDs (Laser Components COUNT-250B). All APD signals were recorded

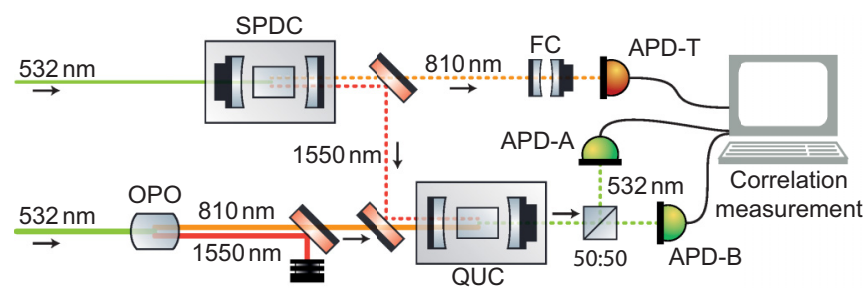

FIG. 1. (Color online) Experimental setup [31]. See text for details. 
with an oscilloscope (Agilent DSO7014A). More details about the experimental setup can be found in Refs. [31,34,35].

A click of the trigger detector APD-T at time $t_{0}$ heralds the preparation of a single-photon state in the up-converted signal beam at $532 \mathrm{~nm}$, which is characterized by a HanburyBrown-Twiss scheme. We introduce a coincidence window of total width $\Delta t$ centered on $t_{0}$ and count the number of events $R_{1 A}\left(R_{1 B}\right)$ when a click of APD-A (APD-B) occurs within the coincidence window while the other detector APD-B (APD-A) does not click. We also count the number of events $R_{2}$ when both APD-A and APD-B click within the coincidence window, and the total number of clicks of the trigger detector $R_{T}$. Due to losses, noise, and multiple-pair contributions the conditionally generated state of the signal beam is a mixture of a vacuum and single-photon state, with a small fraction of multiphoton terms.

While the measured coincidences do not provide enough data to completely determine the photon number statistics of the conditionally generated state, they are sufficient to estimate the vacuum and single-photon probabilities, which are most relevant in this case. As shown in Ref. [33], the vacuum probability $p_{0}$ is given by

$$
p_{0}=1-\frac{R_{1 A}+R_{1 B}+R_{2}}{R_{T}},
$$

while the single-photon probability $p_{1}$ can be lower bounded as follows,

$$
p_{1} \geqslant \frac{R_{1 A}+R_{1 B}}{R_{T}}-\frac{T^{2}+(1-T)^{2}}{2 T(1-T)} \frac{R_{2}}{R_{T}} .
$$

Here $T$ denotes the transmittance of the beam splitter in Hanbury-Brown-Twiss scheme, which can be estimated from the measured coincidences as $T=R_{1 A} /\left(R_{1 A}+R_{1 B}\right)$. We found that $T \approx 0.525$, which is close to the nominal value of $50 \%$. We note that the lower bound (6) coincides with the true value of $p_{1}$ if the state does not contain more than two photons. The measurement was performed for three different values of parametric gain of the SPDC cavity $\epsilon=\gamma \sqrt{P / P_{\mathrm{th}}}$, where $P_{\text {th }}$ denotes the threshold pump power for parametric oscillation, and $\gamma=\pi \times 31 \mathrm{MHz}$ is the mean decay rate of the SPDC cavity. The experimental results are presented in Sec. VI where they are compared with predictions of the theoretical model.

\section{SINGLE-MODE MODEL}

Here we describe a simple theoretical model of the experiment where each beam is treated as a single mode. We assume that the signal and idler modes are initially prepared in a pure two-mode squeezed vacuum state

$$
\left|\psi_{\mathrm{TMSV}}\right\rangle_{S I}=\sqrt{1-\sigma^{2}} \sum_{n=0}^{\infty} \sigma^{n}|n, n\rangle_{S I},
$$

where $\sigma=\tanh r$ and $r$ denotes the squeezing constant. This two-mode Gaussian state can be conveniently described by a
$4 \times 4$ covariance matrix

$\gamma_{S I, 0}=\left(\begin{array}{cccc}\cosh (2 r) & 0 & \sinh (2 r) & 0 \\ 0 & \cosh (2 r) & 0 & -\sinh (2 r) \\ \sinh (2 r) & 0 & \cosh (2 r) & 0 \\ 0 & -\sinh (2 r) & 0 & \cosh (2 r)\end{array}\right)$,

whose elements represent variances and covariances of quadrature operators, $\left(\gamma_{S I}\right)_{j k}=\left\langle\Delta q_{j} \Delta q_{k}+\Delta q_{k} \Delta q_{j}\right\rangle, q=$ $\left(x_{S}, p_{S}, x_{I}, p_{I}\right), \Delta q_{j}=q_{j}-\left\langle q_{j}\right\rangle$, and the quadrature operators are defined in terms of annihilation and creation operators $a_{j}$ and $a_{j}^{\dagger}$ as

$$
x_{j}=\frac{a_{j}+a_{j}^{\dagger}}{\sqrt{2}}, \quad p_{j}=\frac{a_{j}-a_{j}^{\dagger}}{i \sqrt{2}} .
$$

The signal and idler modes propagate through lossy quantum channels with transmittances $\eta_{S}$ and $\eta_{I}$, respectively. These transmittances account for all losses and inefficiencies in the setup including detection efficiencies of the single-photon detectors and a limited efficiency of the quantum up-conversion of signal mode. At the output of the lossy channels, the two-mode covariance matrix reads

$$
\gamma_{S I}=S \gamma_{S I, 0} S^{T}+G
$$

where

$$
S=\left(\begin{array}{cccc}
\sqrt{\eta_{S}} & 0 & 0 & 0 \\
0 & \sqrt{\eta_{S}} & 0 & 0 \\
0 & 0 & \sqrt{\eta_{I}} & 0 \\
0 & 0 & 0 & \sqrt{\eta_{I}}
\end{array}\right)
$$

and

$$
G=\left(\begin{array}{cccc}
1-\eta_{S} & 0 & 0 & 0 \\
0 & 1-\eta_{S} & 0 & 0 \\
0 & 0 & 1-\eta_{I} & 0 \\
0 & 0 & 0 & 1-\eta_{I}
\end{array}\right) .
$$

The signal mode is analyzed with the help of the HanburyBrown-Twiss setup where it is combined with an auxiliary vacuum mode on a balanced beam splitter and the output modes A and $\mathrm{B}$ are observed with single-photon detectors APD-A and APD-B. The photodetectors are avalanche photodiodes that can respond with two outcomes: either a click or a no click. We model these detectors by the following two-component POVM,

$$
\Pi_{1}=I-|0\rangle\left\langle 0\left|, \quad \Pi_{0}=\right| 0\right\rangle\langle 0|,
$$

where $I$ denotes the identity operator and $|0\rangle$ is the vacuum state. Note that the detector efficiencies are incorporated in the overall effective transmittances $\eta_{j}$, that were introduced above, and we neglect other effects such as dark counts.

We will calculate the probabilities of various single and coincidence clicks using the complementary probabilities of projections onto vacuum. Consider an $N$-mode Gaussian state $\rho_{N}$ with covariance matrix $\gamma_{N}$ and vanishing coherent displacement. Then it can be shown using the Husimi $Q$ function formalism that the overlap of this state with an $N$-mode vacuum state reads

$$
\left\langle 0, \ldots, 0\left|\rho_{N}\right| 0, \ldots, 0\right\rangle=\frac{2^{N}}{\sqrt{\operatorname{det} \gamma_{N}^{Q}}},
$$


where $\gamma_{N}^{Q}=\gamma_{N}+I$ is the covariance matrix corresponding to the antinormal ordering of operators and $I$ denotes the identity matrix. We define

$$
\gamma_{S I}^{Q}=\gamma_{S I}+I
$$

The probability $R_{T}$ of a click of the trigger detector APD-T can be expressed as

$$
R_{T}=\operatorname{Tr}\left[\rho_{S I} I_{S} \otimes \Pi_{1, I}\right]=1-\frac{2}{\sqrt{\operatorname{det} \gamma_{I}^{Q}}},
$$

where $\gamma_{I}^{Q}$ denotes the single-mode submatrix of $\gamma_{S I}^{Q}$ corresponding to the idler mode, and we also define $\gamma_{S}^{Q}$ in a similar way. Let $P_{0, A B}$ denote the probability that APD-T clicks while none of the detectors APD-A and APD-B click. This is equivalent to the probability that the idler mode is projected onto $\Pi_{1}$ while the signal mode is projected onto vacuum,

$$
P_{0, A B}=\operatorname{Tr}\left[\rho_{S I} \Pi_{0, S} \otimes \Pi_{1, I}\right] .
$$

With the use of Eqs. (13) and (14) we get

$$
P_{0, A B}=\frac{2}{\sqrt{\operatorname{det} \gamma_{S}^{Q}}}-\frac{4}{\sqrt{\operatorname{det} \gamma_{S I}^{Q}}} .
$$

A final ingredient that we need is the probability $P_{0, A}$ that detector APD-T clicks and detector APD-A does not click irrespective of the response of detector APD-B. In order to calculate this probability we can treat the balanced beam splitter that splits the signal between APD-A and APD-B as an additional lossy channel with transmittance $1 / 2$. We introduce a new overall effective transmittance for the signal beam $\tilde{\eta}_{S}=\eta_{S} / 2$ and determine covariance matrices $\tilde{\gamma}_{S I}^{Q}$ and $\tilde{\gamma}_{S}^{Q}$ corresponding to these modified losses of the signal beam. We then have

$$
P_{0, A}=\frac{2}{\sqrt{\operatorname{det} \tilde{\gamma}_{S}^{Q}}}-\frac{4}{\sqrt{\operatorname{det} \tilde{\gamma}_{S I}^{Q}}} .
$$

In what follows, we denote by $R_{1 A}$ the probability that APD-T and APD-A click while APD-B does not click, $R_{1 B}$ is defined analogously, and $R_{2}$ represents the probability of threefold coincidence clicks of all three detectors. The probabilities $P_{0, A B}$ and $P_{0, A}$ can then be expressed as follows,

$$
\begin{aligned}
P_{0, A} & =R_{T}-R_{1 A}-R_{2}, \\
P_{0, A B} & =R_{T}-R_{1 A}-R_{1 B}-R_{2} .
\end{aligned}
$$

Since in our theoretical model we assume symmetric detection with a balanced beam splitter, we have $R_{1 A}=R_{1 B}$ and we obtain

$$
\begin{aligned}
R_{1 A} & =P_{0, A}-P_{0, A B}, \\
R_{2} & =R_{T}-2 P_{0, A}+P_{0, A B} .
\end{aligned}
$$

The above formulas allow us to determine $R_{j}$ for any squeezing constant $r$ and overall effective transmittances $\eta_{S}$ and $\eta_{I}$. The probabilities $p_{0}$ and $p_{1}$ of a vacuum and singlephoton state can then be estimated using Eqs. (5) and (6), where we need to set $T=1 / 2$ since our model assumes a perfectly balanced beam splitter. The results are plotted in Fig. 2 for $\eta_{S}=0.20$ and $\eta_{I}=0.11$, which are the estimated values of

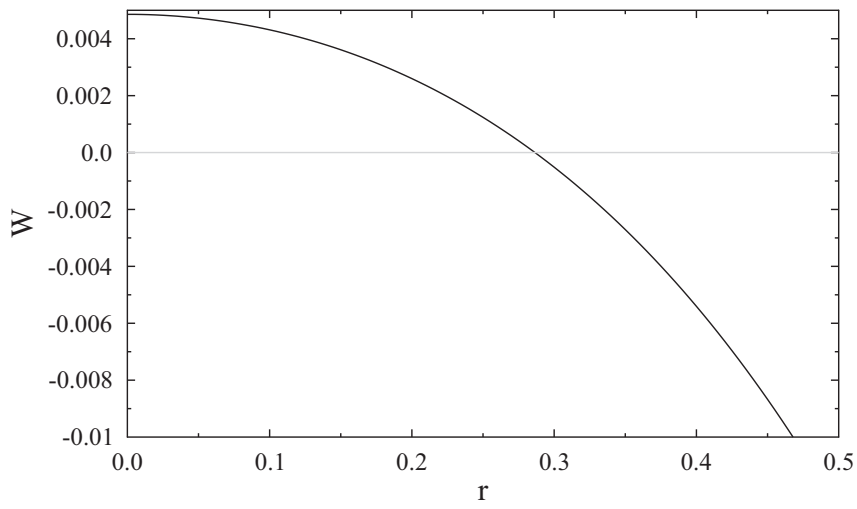

FIG. 2. The quantum non-Gaussianity witness $W$ is plotted as a function of squeezing constant $r$ for $\eta_{S}=0.20$ and $\eta_{I}=0.11$.

the overall effective transmittances in our experiment. The plot shows that $W$ is a monotonically decreasing function of the squeezing strength $r$. In the limit $r \rightarrow 0$ the conditionally prepared state reads

$$
\rho_{S}=\eta_{S}|1\rangle\left\langle 1\left|+\left(1-\eta_{S}\right)\right| 0\right\rangle\langle 0|,
$$

and its quantum non-Gaussianity is optimally certified by the witness (1). As the squeezing strength increases, the contribution of multiphoton terms becomes non-negligible, the state deviates from a mixture of vacuum and single photons, and for strong enough squeezing the state cannot be certified as quantum non-Gaussian by witness (1).

We note that instead of witness (1) one can also use a witness formulated directly in terms of the probabilities of clicks and no clicks of detectors APD-A and APD-B [39]. This latter witness allows the detection of a slightly larger class of quantum non-Gaussian states, because the approximation in estimation of $p_{1}$ is avoided, but the results are qualitatively similar for both witnesses. Here we stick to witness (1) because it was employed in our previous work [31] and it can be efficiently calculated by numerically solving Eq. (4).

\section{MULTIMODE MODEL}

Here we present a more refined model that takes into account the temporal structure of quantum correlations between signal and idler beams. Quantum statistical properties of $\mathrm{cw}$ squeezed light generated in the SPDC cavity are fully specified by second-order temporal correlation functions [45]

$$
\begin{aligned}
& \left\langle a_{-}^{\dagger}\left(t_{1}\right) a_{-}\left(t_{2}\right)\right\rangle=\left\langle a_{+}^{\dagger}\left(t_{1}\right) a_{+}\left(t_{2}\right)\right\rangle=\mathcal{N}\left(t_{1}-t_{2}\right), \\
& \left\langle a_{-}\left(t_{1}\right) a_{+}\left(t_{2}\right)\right\rangle=\mathcal{M}\left(t_{1}-t_{2}\right),
\end{aligned}
$$

where $a_{-}(t)$ and $a_{+}(t)$ denote annihilation operators of signal and idler beams, respectively,

$$
\begin{aligned}
& \mathcal{N}(\tau)=\frac{\lambda^{2}-\mu^{2}}{4}\left(\frac{e^{-\mu|\tau|}}{2 \mu}-\frac{e^{-\lambda|\tau|}}{2 \lambda}\right), \\
& \mathcal{M}(\tau)=\frac{\lambda^{2}-\mu^{2}}{4}\left(\frac{e^{-\mu|\tau|}}{2 \mu}+\frac{e^{-\lambda|\tau|}}{2 \lambda}\right),
\end{aligned}
$$

$\lambda=\gamma+\epsilon$ and $\mu=\gamma-\epsilon$. Since the overall quantum state of the optical field is Gaussian, higher-order correlations 
and moments can be expressed in terms of the second-order correlations $\mathcal{M}(\tau)$ and $\mathcal{N}(\tau)$.

The signal beam experiences an additional filtering due to a slightly smaller SPDC cavity decay rate compared to the idler beam and transmission through the quantum up-conversion cavity. This additional filtering can be modeled by a single filter cavity with bandwidth $\kappa$ that acts on the signal beam $a_{-}(t)$ only. The transformed annihilation operator of the signal beam reads,

$$
a_{-}^{\prime}(t)=\kappa \int_{-\infty}^{t} e^{-\kappa(t-y)} a_{-}(y) d y+\xi(t),
$$

where $\xi(t)$ represents the vacuum noise necessary for the preservation of canonical commutation relations. Since $\xi(t)$ does not contribute to normally ordered correlation functions, it can be neglected in subsequent calculations. The correlation functions

$$
\begin{aligned}
& \mathcal{N}^{\prime}\left(t_{1}-t_{2}\right)=\left\langle a_{-}^{\prime \dagger}\left(t_{1}\right) a_{-}^{\prime}\left(t_{2}\right)\right\rangle, \\
& \mathcal{M}^{\prime}\left(t_{1}-t_{2}\right)=\left\langle a_{-}^{\prime}\left(t_{1}\right) a_{+}\left(t_{2}\right)\right\rangle,
\end{aligned}
$$

can be determined as integrals of the original correlation functions (24),

$$
\mathcal{M}^{\prime}\left(t_{1}-t_{2}\right)=\kappa \int_{-\infty}^{t_{1}} e^{-\kappa\left(t_{1}-y\right)} \mathcal{M}\left(y-t_{2}\right) d y,
$$

and

$$
\mathcal{N}^{\prime}\left(t_{1}-t_{2}\right)=\kappa^{2} \int_{-\infty}^{t_{1}} \int_{-\infty}^{t_{2}} e^{-\kappa\left(t_{1}+t_{2}-y-z\right)} \mathcal{N}(y-z) d y d z
$$

After some algebra, we obtain

$$
\begin{aligned}
\mathcal{N}^{\prime}(\tau)= & \frac{\kappa^{2}\left(\lambda^{2}-\mu^{2}\right)}{4}\left[\frac{e^{-\mu|\tau|}}{2 \mu\left(\kappa^{2}-\mu^{2}\right)}-\frac{e^{-\lambda|\tau|}}{2 \lambda\left(\kappa^{2}-\lambda^{2}\right)}\right. \\
& \left.+\frac{\left(\lambda^{2}-\mu^{2}\right) e^{-\kappa|\tau|}}{2 \kappa\left(\kappa^{2}-\mu^{2}\right)\left(\kappa^{2}-\lambda^{2}\right)}\right], \\
\mathcal{M}^{\prime}(\tau)= & \frac{\lambda^{2}-\mu^{2}}{4}\left[\frac{e^{-\mu|\tau|}}{2 \mu} \frac{\kappa}{\kappa+\mu}+\frac{e^{-\lambda|\tau|}}{2 \lambda} \frac{\kappa}{\kappa+\lambda}\right], \quad \tau<0, \\
\mathcal{M}^{\prime}(\tau)= & \frac{\lambda^{2}-\mu^{2}}{4}\left[\frac{e^{-\mu|\tau|}}{2 \mu} \frac{\kappa}{\kappa-\mu}+\frac{e^{-\lambda|\tau|}}{2 \lambda} \frac{\kappa}{\kappa-\lambda}\right. \\
& \left.+\frac{\kappa\left(\lambda^{2}+\mu^{2}-2 \kappa^{2}\right) e^{-\kappa|\tau|}}{\left(\kappa^{2}-\lambda^{2}\right)\left(\kappa^{2}-\mu^{2}\right)}\right], \quad \tau \geqslant 0 .
\end{aligned}
$$

The losses due to filtering (25) can be estimated by introducing an effective transmittance of the filter cavity $\eta_{\kappa}=$ $\mathcal{N}^{\prime}(0) / \mathcal{N}(0)$. After some algebra we obtain

$$
\eta_{\kappa}=\frac{\kappa(\kappa+2 \gamma)}{(\kappa+\gamma)^{2}-\epsilon^{2}} .
$$

In our experiment, $\kappa=1.4 \gamma$ and we estimate that $\epsilon \leqslant 0.2 \gamma$. The effective transmittance $\eta_{\kappa}$ is a slowly increasing function of $\epsilon$, which is almost constant in the interval $0<\epsilon<0.2 \gamma$. In particular, we have $\eta_{\kappa}=0.826$ for $\epsilon \rightarrow 0$ and $\eta_{\kappa}=0.832$ for $\epsilon=0.2 \gamma$. Since the losses due to filtering are not included in $\eta_{S}$, the overall detection efficiency of the signal beam reads $\eta_{S} \eta_{\kappa}$. We estimate $\eta_{S} \eta_{\kappa} \approx 0.20$, which corresponds to $\eta_{S} \approx 0.24$.

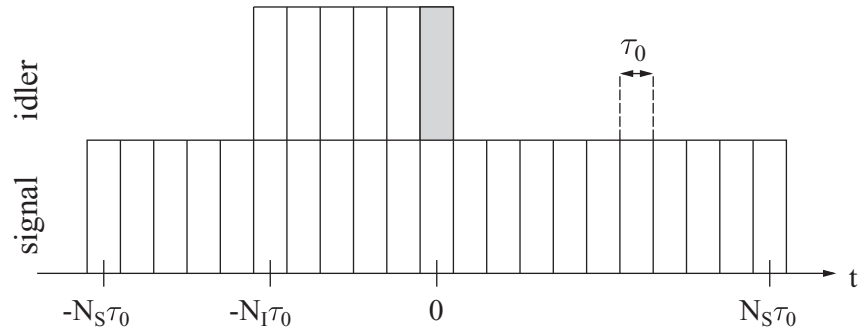

FIG. 3. Discretization of the coincidence window into $2 N_{S}+1$ rectangular modes of width $\tau_{0}$. The trigger detector clicks at time $t_{0}=0$, which is modeled by assuming that APD-T detects a photon belonging to the rectangular temporal mode highlighted by gray color. The figure also shows the rectangular temporal modes of the idler beam that are introduced to account for the dead time of the trigger detector APD-T $t_{D}=N_{I} \tau_{0}$.

Without loss of generality we can assume that the trigger detector APD-T clicks at time $t_{0}=0$, and we introduce a symmetric coincidence window of total width $\Delta t$ for clicks of the detectors APD-A and APD-B. In order to calculate the probabilities of clicks and no clicks of the various detectors and their combinations, we discretize the coincidence window by dividing it into $2 N_{S}+1$ slots of temporal width $\tau_{0}$. We introduce a signal mode with rectangular temporal profile for each slot,

$$
a_{j,-}^{\prime}=\frac{1}{\sqrt{\tau_{0}}} \int_{j \tau_{0}-\frac{\tau_{0}}{2}}^{j \tau_{0}+\frac{\tau_{0}}{2}} a_{-}^{\prime}(t) d t .
$$

This discretization procedure is graphically illustrated in Fig. 3. We also introduce analogous modes for the idler beam,

$$
a_{k,+}=\frac{1}{\sqrt{\tau_{0}}} \int_{k \tau_{0}-\frac{\tau_{0}}{2}}^{k \tau_{0}+\frac{\tau_{0}}{2}} a_{+}(t) d t .
$$

The correlations of discrete modes $a_{j,-}^{\prime}$ and $a_{k,+}$ defined as

$$
\begin{aligned}
& \left\langle a_{j,+}^{\dagger} a_{k,+}\right\rangle=\mathcal{N}_{j k}, \\
& \left\langle a_{j,-}^{\prime \dagger} a_{k,-}^{\prime}\right\rangle=\mathcal{N}_{j k}^{\prime}, \\
& \left\langle a_{j,-}^{\prime} a_{k,+}\right\rangle=\mathcal{M}_{j k},
\end{aligned}
$$

can be straightforwardly calculated using the above definitions. For instance

$$
\mathcal{N}_{j k}=\frac{1}{\tau_{0}} \int_{-\frac{\tau_{0}}{2}}^{\frac{\tau_{0}}{2}} \int_{-\frac{\tau_{0}}{2}}^{\frac{\tau_{0}}{2}} \mathcal{N}\left((j-k) \tau_{0}+t_{1}-t_{2}\right) d t_{1} d t_{2} .
$$

If $j \neq k$, then $(j-k) \tau_{0}+t_{1}-t_{2}$ is either positive or negative for all $t_{1}, t_{2} \in\left[-\tau_{0} / 2, \tau_{0} / 2\right]$ so the $|\tau|$ in the definition of $\mathcal{N}(\tau)$ and $\mathcal{M}(\tau)$ can be replaced with $\tau$ or $-\tau$. However, if $j=k$, then the argument of $\mathcal{N}$ in the integral (34) can be both positive or negative and its evaluation requires special consideration. The required integrations can be performed with the help of the following formula,

$$
\frac{1}{\tau_{0}} \int_{-\frac{\tau_{0}}{2}}^{\frac{\tau_{0}}{2}} \int_{-\frac{\tau_{0}}{2}}^{\frac{\tau_{0}}{2}} e^{-\lambda\left|t_{1}-t_{2}\right|} d t_{1} d t_{2}=\tau_{0} h\left(\lambda \tau_{0}\right),
$$


where

$$
h(x)=\frac{2}{x^{2}}\left(x-1+e^{-x}\right) .
$$

The resulting expressions for $\mathcal{N}_{j k}, \mathcal{N}_{j k}^{\prime}$, and $\mathcal{M}_{j k}^{\prime}$ are rather lengthy and are provided in the Appendix.

The discretization makes the calculations only approximate, because some part of the signal is contained in temporal modes orthogonal to the rectangular modes (31) and (32). However, in the limit $\tau_{0} \rightarrow 0$ all signal becomes contained in the rectangular modes [18]. To quantify the degree of approximation caused by discretization, we compare the total mean number of photons in time interval $\tau_{0}, \bar{n}=\mathcal{N}(0) \tau_{0}$, with the mean number of photons in a single rectangular mode, $\bar{n}_{R}=\mathcal{N}_{00}$. Assuming that $\tau_{0} \gamma \ll 1$ and using the Taylor series expansion $h(x) \approx 1-x / 3+x^{2} / 12$ we find that the fraction of photons contained in other modes is given by

$$
\delta n=\frac{\bar{n}-\bar{n}_{R}}{\bar{n}} \approx \frac{\mu \lambda \tau_{0}^{2}}{12} .
$$

In all subsequent calculations, we set $\tau_{0}=0.5 \mathrm{~ns}$ and $\gamma=$ $\pi \times 31 \mathrm{MHz}$, hence $\gamma \tau_{0}=0.0487 \ll 1$, and for $\epsilon=0.1 \gamma$ we have $\delta n=1.92 \times 10^{-4}$. We have checked that if we further reduce $\tau_{0}$ the numerical results remain unchanged, which confirms sufficient precision of our calculations.

We include in our model the influence of the dead time $t_{D}$ of the trigger detector APD-T. The dead time denotes a time interval after a click of an APD, during which this detector is blind and cannot detect any photons. This means that if APD-T clicks at time $t_{0}=0$ the detector did not detect any photons in the time interval $\left[-t_{D}, 0\right]$, because otherwise it would be blind and could not have detected a photon at time $t_{0}=0$. The fact that the trigger detector did not register any photons in the time interval $\left[-t_{D}, 0\right]$ implies that the idler beam was projected onto vacuum in this time interval [36-38]. To account for this effect, we introduce $N_{I}$ time slots for the idler beam that span the time interval $\left[-t_{D}, 0\right]$ and $N_{I}=t_{D} / \tau_{0}$, see Fig. 3 . A mode with rectangular temporal profile (32) is associated with each time slot and all these $N_{I}$ modes are projected on vacuum. Note that the situation is actually more subtle because if APD-T clicked in time interval $\left[-2 t_{D},-t_{D}\right]$, then it would be blind during some part of the time interval $\left[-t_{D}, 0\right]$. However, the probability of such event is very low for parameters considered and we have verified by explicit calculations that this feature can be neglected.

Our model thus includes in total $2 N_{S}+1$ modes of the signal beam and $N_{I}+1$ modes of the idler beam. With the expressions for the correlations $\mathcal{N}_{j k}, \mathcal{N}_{j k}^{\prime}$, and $\mathcal{M}_{j k}$ at hand, one can construct the $2 N_{S}+N_{I}+2$-mode covariance matrix $\gamma_{S I}$ that fully specifies the Gaussian quantum state of modes $a_{j,-}^{\prime}$ and $a_{k,+}$. In particular,

$$
\begin{aligned}
& \left\langle\Delta x_{j,+} \Delta x_{k,+}\right\rangle=\left\langle\Delta p_{j,+} \Delta p_{k,+}\right\rangle=\frac{\delta_{j k}}{2}+\mathcal{N}_{j k}, \\
& \left\langle\Delta x_{j,-}^{\prime} \Delta x_{k,-}^{\prime}\right\rangle=\left\langle\Delta p_{j,-}^{\prime} \Delta p_{k,-}^{\prime}\right\rangle=\frac{\delta_{j k}}{2}+\mathcal{N}_{j k}^{\prime}, \\
& \left\langle\Delta x_{j,-}^{\prime} \Delta x_{k,+}\right\rangle=-\left\langle\Delta p_{j,-}^{\prime} \Delta p_{k,+}\right\rangle=\mathcal{M}_{j k},
\end{aligned}
$$

and all other correlations vanish.
Similarly to the single-mode model, losses and inefficient detection can be modeled by (multimode) lossy channels with transmittances $\eta_{S}$ and $\eta_{I}$, respectively. Assuming mode ordering where the modes of the signal beam are placed first, we have

$$
\begin{array}{ll}
S_{j j}=\sqrt{\eta_{S}}, & G_{j j}=\sqrt{1-\eta_{S}}, \quad j \leqslant 2\left(2 N_{S}+1\right), \\
S_{j j}=\sqrt{\eta_{I}}, & G_{j j}=\sqrt{1-\eta_{I}}, \quad j>2\left(2 N_{S}+1\right) .
\end{array}
$$

In order to calculate the probabilities of clicks and no clicks of various detection combinations, we first define covariance matrices for several subsets of modes. Let $\gamma_{I}$ denote the covariance matrix of $N_{I}+1$ rectangular temporal modes of the idler beam $a_{k,+},-N_{I} \leqslant k \leqslant 0 . \gamma_{J}$ then represents the covariance matrix of $N_{I}$ modes $a_{k,+}$ with $-N_{I} \leqslant k<0$, i.e., the mode $a_{0,+}$ centered on $t_{0}=0$ is excluded. Finally, $\gamma_{S J}$ denotes a covariance matrix of $2 N_{S}+1$ modes of signal beam $a_{j,-}^{\prime},-N_{S} \leqslant j \leqslant N_{S}$, and $N_{I}$ modes of the idler beam, with mode $a_{0,+}$ centered on $t_{0}=0$ being excluded. The probability of a click of APD-T in a short time window centered at $t_{0}=0$ and having the width $\tau_{0}$ can be expressed as

$$
R_{T}=\frac{2^{N_{I}}}{\sqrt{\operatorname{det} \gamma_{J}^{Q}}}-\frac{2^{N_{I}+1}}{\sqrt{\operatorname{det} \gamma_{I}^{Q}}},
$$

and we recall that the superscript $Q$ denotes covariance matrices corresponding to antinormal operator ordering, $\mathrm{cf}$. Sec. IV. The probability that APD-T clicks and none of the detectors APD-A and APD-B clicks within the coincidence window with width $\Delta t$ reads

$$
P_{0, A B}=\frac{2^{2 N_{S}+N_{I}+1}}{\sqrt{\operatorname{det} \gamma_{S J}^{Q}}}-\frac{2^{2 N_{S}+N_{I}+2}}{\sqrt{\operatorname{det} \gamma_{S I}^{Q}}} .
$$

Similarly as in Sec. IV, we introduce the covariance matrix $\tilde{\gamma}_{S I}$ corresponding to the modified losses of the signal beam, $\tilde{\eta}_{S}=\eta_{S} / 2$. The probability that detector APD-A does not click within the coincidence window irrespective of the response of APD-B then reads

$$
P_{0, A}=\frac{2^{2 N_{S}+N_{I}+1}}{\sqrt{\operatorname{det} \tilde{\gamma}_{S J}^{Q}}}-\frac{2^{2 N_{S}+N_{I}+2}}{\sqrt{\operatorname{det} \tilde{\gamma}_{S I}^{Q}}} .
$$

The probabilities $R_{1 A}, R_{1 B}$ and $R_{2}$ can be calculated using Eq. (21) similarly as for the single-mode model.

\section{RESULTS}

The experimentally determined dependence of the quantum non-Gaussianity witness $W$ on the width of the coincidence window $\Delta t$ is plotted as dots in Fig. 4, where each panel corresponds to a different parametric gain $\epsilon$ of the SPDC cavity. The solid lines represent theoretical fits to the data obtained from our model. We can see that the model correctly reproduces all experimentally observed features of the dependence of $W$ on the coincidence window size and the parametric gain. In our calculations, we fix $\gamma=\pi \times 31 \mathrm{MHz}$ and $t_{D}=29 \mathrm{~ns}$, which were determined by independent measurements, and we set $\kappa=1.4 \gamma$, which was estimated by fitting the temporal profile of the conditionally prepared single-photon state with function $\left|\mathcal{M}^{\prime}(\tau)\right|^{2}$ [31]. The overall detection efficiencies $\eta_{S}$ and $\eta_{I}$ 


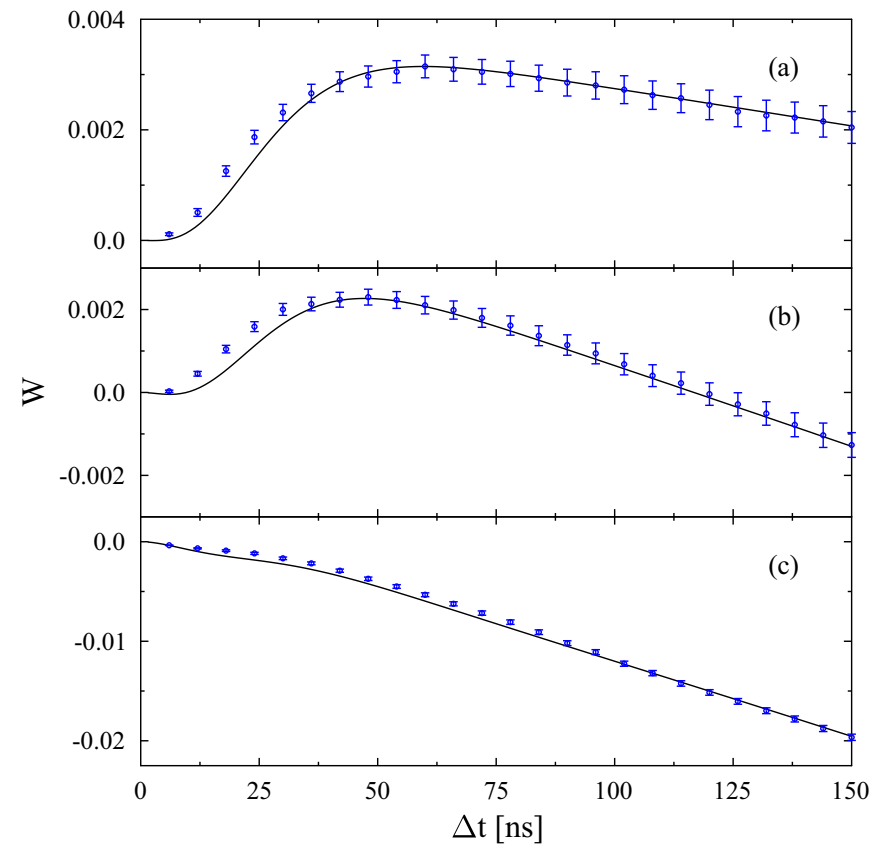

FIG. 4. (Color online) Dependence of the non-Gaussianity witness $W$ on the coincidence window size $\Delta t$. Blue dots represent experimental results for three different pump powers $P$, and solid lines represent predictions of the theoretical model for (a) $\gamma=\pi \times 31 \mathrm{MHz}$, $\eta_{I}=0.11, t_{D}=29 \mathrm{~ns}, \kappa=1.4 \gamma, \eta_{S}=0.235, \epsilon=0.070 \gamma$, (b) $\eta_{S}=$ $0.252, \epsilon=0.109 \gamma$, and (c) $\eta_{S}=0.260, \epsilon=0.194 \gamma$. The error bars were estimated assuming Poissonian statistics of the count rates and they represent one standard deviation.

could be slightly different for each parametric gain $\epsilon$ due to the realignment of the optical setup that was performed before data acquisition for each $\epsilon$. Our calculations show that good fits can be obtained for a wide range of values of $\eta_{I}$. We have therefore estimated $\eta_{I}$ from the observed trigger count rates, which according to our model are given by

$$
\eta_{I} \mathcal{N}(0)=\eta_{I} \frac{\gamma \epsilon^{2}}{\gamma^{2}-\epsilon^{2}} .
$$

The fitting procedure proceeds as follows. We choose an initial value of $\eta_{I}$ and optimize the parameters $\eta_{S}$ and $\epsilon$ to obtain good fits, while keeping $\eta_{S}$ close to 0.24 . The transmittance $\eta_{I}$ is then determined in a self-consistent way from the estimated values of $\epsilon$ and the observed trigger count rates, and we seek new fits for this updated value of $\eta_{I}$. This iterative procedure yields $\eta_{I}=0.11$, and the values of $\eta_{S}$ and $\epsilon$ obtained in this way are summarized in Table I. This table

TABLE I. Parameters $\eta_{S}$ and $\epsilon$ of the optimal fits to the experimental data for three different pump powers $P$. Also shown are the nominal values of the parametric gain $\epsilon_{\text {nom }}$ as reported in our previous experimental work [31].

\begin{tabular}{lccc}
\hline \hline$P$ & $\epsilon_{\text {nom }} / \gamma$ & $\eta_{S}$ & $\epsilon / \gamma$ \\
\hline $0.7 \mathrm{~mW}$ & 0.10 & 0.235 & 0.070 \\
$1.8 \mathrm{~mW}$ & 0.16 & 0.252 & 0.109 \\
$5.5 \mathrm{~mW}$ & 0.28 & 0.260 & 0.194 \\
\hline \hline
\end{tabular}

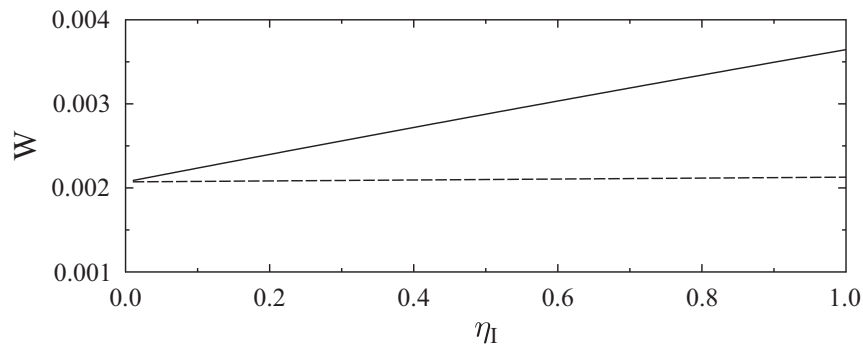

FIG. 5. Dependence of the quantum non-Gaussianity witness $W$ on $\eta_{I}$. The other parameters read $\gamma=\pi \times 31 \mathrm{MHz}, \eta_{S}=0.252$, $t_{D}=29 \mathrm{~ns}, \kappa=1.4 \gamma, \epsilon=0.109 \gamma$, and $\Delta t=50 \mathrm{~ns}$. For comparison, the dashed line indicates results for a detector with $t_{D}=0$.

also lists the nominal values of $\epsilon$ as reported in our previous experimental work [31]. We can see that the parametric gains $\epsilon$ determined by fitting the measured non-Gaussianity witnesses $W$ are consistently smaller than the nominal values reported in Ref. [31]. Previously we determined the ratios of $\epsilon$ by measuring the pump powers $P$, but the threshold power $P_{\text {th }}$ could not be directly determined, which led to some degree of uncertainty in the estimation of the parametric gain. We checked that the present estimates of $\epsilon$ are consistent with the measured pump powers and other experimental data.

The dependence of the non-Gaussianity witness on the overall efficiency of the trigger detector $\eta_{I}$ is shown in Fig. 5. This figure illustrates the importance of the dead time $t_{D}$ of the trigger detector APD-T. The values of the witness predicted for a hypothetical detector with $t_{D}=0$ are practically constant and independent of $\eta_{I}$, while for a detector with $t_{D}=29 \mathrm{~ns}$ the witness linearly increases with $\eta_{I}$. The difference is already noticeable for an inefficient detector with $\eta_{I}=0.1$, and for realistically achievable overall efficiency of $\eta_{I}=0.4$ the difference becomes quite significant. The relatively weak dependence of $W$ on $\eta_{I}$ can be explained by noting that in the limit of weak pumping, $\epsilon \rightarrow 0$, a click of APD-T will herald the preparation of a mixture of vacuum and single-photon states in the signal beam, cf. Eq. (22), and $\eta_{I}$ would only influence the success probability of preparation, but not the form of the prepared state.

To further analyze the influence of the dead time of the trigger detector APD-T, we plot in Fig. 6 the probability $P_{S}=\left(R_{1 A}+R_{1 B}\right) / R_{T}$ of a click of a single detector APD-A or APD-B in the coincidence window, and probability $P_{C}=$ $R_{2} / R_{T}$ of simultaneous clicks of both APD-A and APD-B in the coincidence window. We can see that both $P_{S}$ and $P_{C}$ decrease with $t_{D}$ until they reach an asymptotic value. The relative reduction of $P_{C}$ is much larger than the relative reduction of $P_{S}$, which suggests that the projection onto vacuum by APD-T in the time interval $\left[-t_{D}, 0\right]$ suppresses the multiphoton content of the conditionally generated state of the signal beam. This makes the conditionally generated state more suitable for recognition as quantum non-Gaussian by the witness that we employ.

For completeness, Fig. 7 illustrates the dependence of $W$ on $\eta_{S}$. We can see that $W$ strongly depends on $\eta_{S}$ and for low enough $\eta_{S}$ the quantum non-Gaussianity of the generated state cannot be certified by the witness. Our calculations show that the dependence of $W$ on $\kappa$ is qualitatively similar to the 

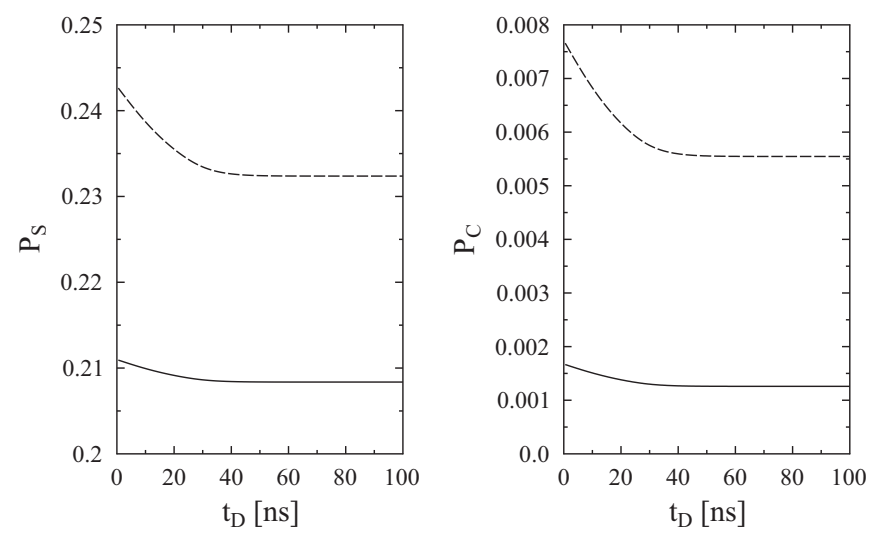

FIG. 6. Dependence of probabilities $P_{S}$ and $P_{C}$ on the dead time of the trigger detector $t_{D}$. The other parameters read $\gamma=\pi \times 31 \mathrm{MHz}$, $\eta_{S}=0.25, \eta_{I}=0.4, \kappa=1.4 \gamma, \Delta t=50 \mathrm{~ns}, \epsilon=0.1 \gamma$ (solid line) and $\epsilon=0.2 \gamma$ (dashed line).

dependence on $\eta_{S}$ because the effective filtering of the signal mode described by Eq. (25) amounts to frequency-dependent losses.

Finally, we note that the measured coincidence counts are influenced by dark counts and after-pulses of the single-photon detectors, and also by the fact that APD-T can be triggered by idler photons at sidebands that are separated in frequency from the carrier by a multiple of a free spectral range of the SPDC cavity, and not completely filtered out by the FC cavity. In case of unwanted trigger events due to dark counts, afterpulses, or uncorrelated idler photons, the signal beam remains in a thermal-like state that is very close to vacuum for the coincidence windows of the order of a few tens of nanoseconds. The unwanted trigger events thus effectively increase losses of the signal beam and the value of $\eta_{S}$ used in our calculations accounts for these effects.

We have found that a part of unwanted trigger events due to after-pulses may be suppressed by introducing a condition that a click of the trigger detector APD-T is accepted as a heralding event if and only if its distance from a previous click of APD-T is at least $t_{\min }$. An after-pulse is a fake click

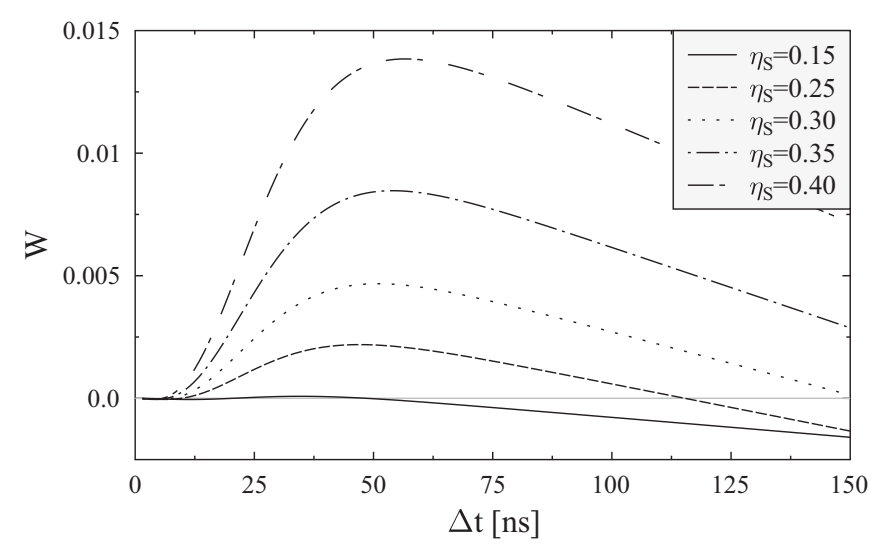

FIG. 7. Dependence of the quantum non-Gaussianity witness $W$ on $\eta_{S}$. The other parameters read $\gamma=\pi \times 31 \mathrm{MHz}, \eta_{I}=0.11$, $t_{D}=29 \mathrm{~ns}, \kappa=1.4 \gamma$, and $\epsilon=0.109 \gamma$.

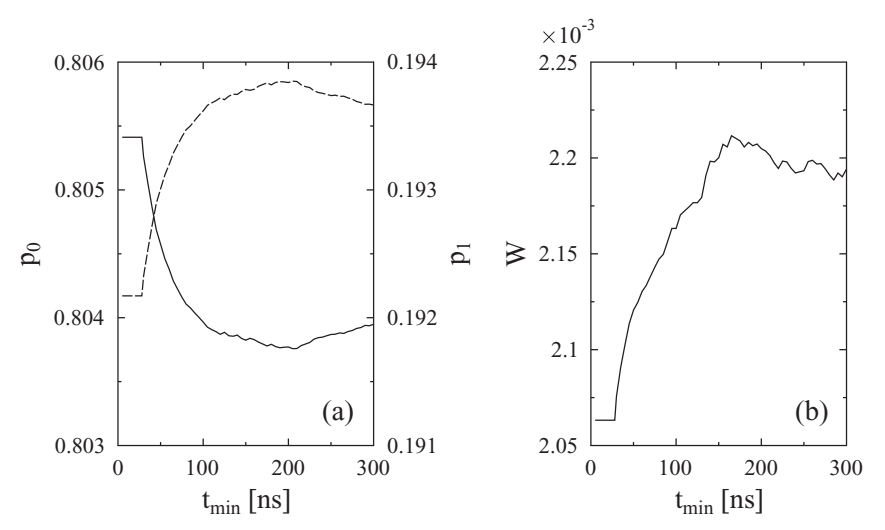

FIG. 8. Experimentally determined dependence of $p_{0}$ (solid line), $p_{1}$ (dashed line), and $W$ on $t_{\min }$. The results are shown for $\epsilon_{\text {nom }}=0.16 \gamma$.

that occurs shortly after a true click of the detector. For APD$\mathrm{T}$ the total probability of an after-pulse is $1 \%$ and the time delay between the initiating click and an after-pulse exhibits exponentially decaying distribution with mean decay time of 28 ns. The experimental results plotted in Fig. 8 show the dependence of $p_{0}, p_{1}$, and $W$ on $t_{\min }$. We can observe that $p_{1}$ increases with $t_{\min }$ while $p_{0}$ decreases, which is consistent with the suppression of false trigger events. Consequently, the quantum non-Gaussianity witness $W$ also increases with $t_{\min }$.

\section{CONCLUSIONS}

In conclusion, we have presented a detailed theoretical description of photon counting measurements on frequency up-converted single-photon states that were conditionally prepared by the detection of idler photons from correlated photon pairs generated in the process of cavity-enhanced spontaneous parametric down-conversion. Our theoretical model fully accounts for the temporal structure of quantum correlations between the signal and idler beams emitted from the SPDC cavity, and its predictions are in very good agreement with the experimental data. The quantum up-conversion is modeled as a lossy channel where part of the losses is assumed to be frequency dependent, which reflects the properties of the up-conversion cavity. In our study, we have focused on a quantum non-Gaussianity witness that depicts the highly nonclassical character of the up-converted state. We employed a witness based on estimates of the vacuum and singlephoton probabilities that were determined from coincidence measurements. Our theoretical calculations confirmed that the maximum observable witness decreases with increasing parametric gain and that there exists an optimal size of the coincidence window leading to the maximum witness. Since the temporal width of the conditionally generated single-photon state is of the order of $1 / \gamma \approx 10 \mathrm{~ns}$, too short coincidence window results in additional losses, as parts of the wave packet fall outside the window. On the other hand, the mean number of thermal-like photons in the signal beam increases with the coincidence window size, and this background noise spoils the quality of the conditionally generated single-photon state if the window is too large. 
Our theoretical analysis also revealed interesting physical phenomena associated with the dead time $t_{D}$ of the trigger detector. A click of the trigger detector at some time $t_{0}$ indicates that the optical beam measured by this detector was projected onto a vacuum state during the preceding time interval $\left[t_{0}-t_{D}, t_{0}\right]$ [36-38]. This effect influences the observed coincidence counts, reduces the multiphoton contribution in the conditionally generated single-photon state, and increases the value of the non-Gaussianity witness. We have also demonstrated that false trigger events due to after-pulses may be partially eliminated by accepting a click of the trigger detector as a heralding event only if its distance from a previous click exceeds a certain threshold. Our theoretical analysis is by no means limited to the particular experimental scheme considered in this paper and the present approach is applicable to a broad class of photon counting experiments with continuous-wave squeezed light.

\section{ACKNOWLEDGMENTS}

This work was supported by the Deutsche Forschungsgemeinschaft (DFG), Project No. SCHN 757/4-1, by the Centre for Quantum Engineering and Space-Time Research (QUEST), and by the International Max Planck Research School for Gravitational Wave Astronomy (IMPRS-GW). J.F. acknowledges financial support from the EU FP7 under Grant Agreement No. 308803 (project BRISQ2) cofinanced by MSMT CR (7E13032).

\section{APPENDIX: CORRELATION COEFFICIENTS}

Here we provide analytical expressions for the correlations $\mathcal{N}_{j k}, \mathcal{N}_{j k}^{\prime}$, and $\mathcal{M}_{j k}$ defined in Eq. (33) of the main text,

$$
\begin{aligned}
& \mathcal{N}_{j k}=\left(\lambda^{2}-\mu^{2}\right)\left[e^{-|j-k| \mu \tau_{0}} \frac{\sinh ^{2}\left(\mu \tau_{0} / 2\right)}{2 \tau_{0} \mu^{3}}-e^{-|j-k| \lambda \tau_{0}} \frac{\sinh ^{2}\left(\lambda \tau_{0} / 2\right)}{2 \tau_{0} \lambda^{3}}\right], \quad j \neq k, \\
& \mathcal{N}_{j j}=\frac{\tau_{0}\left(\lambda^{2}-\mu^{2}\right)}{4}\left[\frac{h\left(\mu \tau_{0}\right)}{2 \mu}-\frac{h\left(\lambda \tau_{0}\right)}{2 \lambda}\right] \text {, } \\
& \mathcal{N}_{j k}^{\prime}=\left(\lambda^{2}-\mu^{2}\right)\left[e^{-|j-k| \mu \tau_{0}} \frac{\sinh ^{2}\left(\mu \tau_{0} / 2\right)}{2 \tau_{0} \mu^{3}} \frac{\kappa^{2}}{\kappa^{2}-\mu^{2}}-e^{-|j-k| \lambda \tau_{0}} \frac{\sinh ^{2}\left(\lambda \tau_{0} / 2\right)}{2 \tau_{0} \lambda^{3}} \frac{\kappa^{2}}{\kappa^{2}-\lambda^{2}}\right. \\
& \left.+e^{-|j-k| \kappa \tau_{0}} \frac{\sinh ^{2}\left(\kappa \tau_{0} / 2\right)}{2 \tau_{0} \kappa^{3}} \frac{\kappa^{2}\left(\lambda^{2}-\mu^{2}\right)}{\left(\kappa^{2}-\mu^{2}\right)\left(\kappa^{2}-\lambda^{2}\right)}\right] j \neq k, \\
& \mathcal{N}_{j j}^{\prime}=\frac{\tau_{0}\left(\lambda^{2}-\mu^{2}\right)}{4}\left[\frac{h\left(\mu \tau_{0}\right)}{2 \mu} \frac{\kappa^{2}}{\kappa^{2}-\mu^{2}}-\frac{h\left(\lambda \tau_{0}\right)}{2 \lambda} \frac{\kappa^{2}}{\kappa^{2}-\lambda^{2}}+\frac{h\left(\kappa \tau_{0}\right)}{2 \kappa} \frac{\kappa^{2}\left(\lambda^{2}-\mu^{2}\right)}{\left(\kappa^{2}-\mu^{2}\right)\left(\kappa^{2}-\lambda^{2}\right)}\right] \text {, } \\
& \mathcal{M}_{j k}=\left(\lambda^{2}-\mu^{2}\right)\left[e^{-|j-k| \mu \tau_{0}} \frac{\sinh ^{2}\left(\mu \tau_{0} / 2\right)}{2 \tau_{0} \mu^{3}} \frac{\kappa}{\kappa+\mu}+e^{-|j-k| \lambda \tau_{0}} \frac{\sinh ^{2}\left(\lambda \tau_{0} / 2\right)}{2 \tau_{0} \lambda^{3}} \frac{\kappa}{\kappa+\lambda}\right], \quad j<k \text {, } \\
& \mathcal{M}_{j k}=\left(\lambda^{2}-\mu^{2}\right)\left[e^{-|j-k| \mu \tau_{0}} \frac{\sinh ^{2}\left(\mu \tau_{0} / 2\right)}{2 \tau_{0} \mu^{3}} \frac{\kappa}{\kappa-\mu}+e^{-|j-k| \lambda \tau_{0}} \frac{\sinh ^{2}\left(\lambda \tau_{0} / 2\right)}{2 \tau_{0} \lambda^{3}} \frac{\kappa}{\kappa-\lambda}\right. \\
& \left.+e^{-|j-k| \kappa \tau_{0}} \frac{\sinh ^{2}\left(\kappa \tau_{0} / 2\right)}{\tau_{0} \kappa^{3}} \frac{\kappa^{2}\left(\lambda^{2}+\mu^{2}-2 \kappa^{2}\right)}{\left(\kappa^{2}-\mu^{2}\right)\left(\kappa^{2}-\lambda^{2}\right)}\right], \quad j>k, \\
& \mathcal{M}_{j j}=\frac{\tau_{0}\left(\lambda^{2}-\mu^{2}\right)}{4}\left[\frac{h\left(\mu \tau_{0}\right)}{2 \mu} \frac{\kappa^{2}}{\kappa^{2}-\mu^{2}}+\frac{h\left(\lambda \tau_{0}\right)}{2 \lambda} \frac{\kappa^{2}}{\kappa^{2}-\lambda^{2}}+\frac{h\left(\kappa \tau_{0}\right)}{2 \kappa} \frac{\kappa^{2}\left(\lambda^{2}+\mu^{2}-2 \kappa^{2}\right)}{\left(\kappa^{2}-\mu^{2}\right)\left(\kappa^{2}-\lambda^{2}\right)}\right] .
\end{aligned}
$$

[1] C. K. Hong and L. Mandel, Phys. Rev. Lett. 56, 58 (1986).

[2] P. Grangier, G. Roger, and A. Aspect, Europhys. Lett. 1, 173 (1986).

[3] A. I. Lvovsky, H. Hansen, T. Aichele, O. Benson, J. Mlynek, and S. Schiller, Phys. Rev. Lett. 87, 050402 (2001).

[4] J. S. Neergaard-Nielsen, B. M. Nielsen, H. Takahashi, A. I. Vistnes, and E. S. Polzik, Opt. Express 15, 7940 (2007).

[5] A. Ourjoumtsev, R. Tualle-Brouri, J. Laurat, and P. Grangier, Science 312, 83 (2006).

[6] J. S. Neergaard-Nielsen, B. M. Nielsen, C. Hettich, K. Mølmer, and E. S. Polzik, Phys. Rev. Lett. 97, 083604 (2006).

[7] K. Wakui, H. Takahashi, A. Furusawa, and M. Sasaki, Opt. Express 15, 3568 (2007).
[8] J. S. Neergaard-Nielsen, M. Takeuchi, K. Wakui, H. Takahashi, K. Hayasaka, M. Takeoka, and M. Sasaki, Phys. Rev. Lett. 105, 053602 (2010).

[9] G. Y. Xiang, T. C. Ralph, A. P. Lund, N. Walk, and G. J. Pryde, Nat. Photon. 4, 316 (2010).

[10] F. Ferreyrol, M. Barbieri, R. Blandino, S. Fossier, R. Tualle-Brouri, and P. Grangier, Phys. Rev. Lett. 104, 123603 (2010).

[11] M. A. Usuga, C. R. Müller, C. Wittmann, P. Marek, R. Filip, C. Marquardt, G. Leuchs, and U. L. Andersen, Nat. Phys. 6, 767 (2010).

[12] A. Zavatta, J. Fiurášek, and M. Bellini, Nat. Photon. 5, 52 (2011). 
[13] H. Takahashi, J. S. Neergaard-Nielsen, M. Takeuchi, M. Takeoka, K. Hayasaka, A. Furusawa, and M. Sasaki, Nature Photon. 4, 178 (2010).

[14] Y. Kurochkin, A. S. Prasad, and A. I. Lvovsky, Phys. Rev. Lett. 112, 070402 (2014).

[15] V. Parigi, A. Zavatta, M. S. Kim, and M. Bellini, Science 317, 1890 (2007).

[16] M. Yukawa, K. Miyata, H. Yonezawa, P. Marek, R. Filip, and A. Furusawa, Phys. Rev. A 88, 053816 (2013).

[17] K. Mølmer, Phys. Rev. A 73, 063804 (2006).

[18] M. Sasaki and S. Suzuki, Phys. Rev. A 73, 043807 (2006).

[19] A. E. B. Nielsen and K. Mølmer, Phys. Rev. A 75, 023806 (2007).

[20] A. E. B. Nielsen and K. Mølmer, Phys. Rev. A 75, 043801 (2007).

[21] A. E. B. Nielsen and K. Mølmer, Phys. Rev. A 75, 063803 (2007).

[22] H. Takahashi, K. Wakui, S. Suzuki, M. Takeoka, K. Hayasaka, A. Furusawa, and M. Sasaki, Phys. Rev. Lett. 101, 233605 (2008).

[23] M. Sasaki, M. Takeoka, and H. Takahashi, Phys. Rev. A 77, 063840 (2008).

[24] P. Kumar, Opt. Lett. 15, 1476 (1990).

[25] J. Huang and P. Kumar, Phys. Rev. Lett. 68, 2153 (1992).

[26] M. A. Albota and F. N. C. Wong, Opt. Lett. 29, 1449 (2004).

[27] S. Tanzilli, W. Tittel, M. Halder, O. Alibart, P. Baldi, N. Gisin, and H. Zbinden, Nature (London) 437, 116 (2005).

[28] C. Langrock, E. Diamanti, R. V. Roussev, Y. Yamamoto, M. M. Fejer, and H. Takesue, Opt. Lett. 30, 1725 (2005).

[29] H. Pan, H. Dong, H. Zeng, and W. Lu, Appl. Phys. Lett. 89, 191108 (2006).
[30] M. T. Rakher, L. Ma, O. Slattery, X. Tang, and K. Srinivasan, Nat. Photon. 4, 786 (2010).

[31] C. Baune, A. Schönbeck, A. Samblowski, J. Fiurášek, and R. Schnabel, Opt. Express 22, 22808 (2014).

[32] R. Filip and L. Mišta, Phys. Rev. Lett. 106, 200401 (2011).

[33] M. Ježek, I. Straka, M. Mičuda, M. Dušek, J. Fiurášek, and R. Filip, Phys. Rev. Lett. 107, 213602 (2011).

[34] C. E. Vollmer, C. Baune, A. Samblowski, T. Eberle, V. Händchen, J. Fiurášek, and R. Schnabel, Phys. Rev. Lett. 112, 073602 (2014).

[35] A. Samblowski, C. E. Vollmer, C. Baune, J. Fiurášek, and R. Schnabel, Opt. Lett. 39, 2979 (2014).

[36] F. Davidson and L. Mandel, J. Appl. Phys. 39, 62 (1968).

[37] F. Davidson, Phys. Rev. 185, 446 (1969).

[38] S. Friberg, J. Andersen, and L. Mandel, Rev. Sci. Instrum. 53, 205 (1982).

[39] L. Lachman and R. Filip, Phys. Rev. A 88, 063841 (2013).

[40] M. G. Genoni, M. L. Palma, T. Tufarelli, S. Olivares, M. S. Kim, and M. G. A. Paris, Phys. Rev. A 87, 062104 (2013).

[41] C. Hughes, M. G. Genoni, T. Tufarelli, M. G. A. Paris, and M. S. Kim, Phys. Rev. A 90, 013810 (2014).

[42] M. Ježek, A. Tipsmark, R. Dong, J. Fiurášek, L. Mišta, R. Filip, and U. L. Andersen, Phys. Rev. A 86, 043813 (2012).

[43] A. Predojević, M. Ježek, T. Huber, H. Jayakumar, T. Kauten, G. S. Solomon, R. Filip, and G. Weihs, Opt. Express 22, 4789 (2014).

[44] I. Straka, A. Predojević, T. Huber, L. Lachman, L. Butschek, M. Miková, M. Mičuda, G. S. Solomon, G. Weihs, M. Ježek, and R. Filip, Phys. Rev. Lett. 113, 223603 (2014).

[45] P. D. Drummond and M. D. Reid, Phys. Rev. A 41, 3930 (1990). 